\title{
Comparative sequence analysis for Brassica oleracea with similar sequences in B. rapa and Arabidopsis thaliana
}

\author{
Dan Qiu · Muqiang Gao · Genyi Li · \\ Carlos Quiros
}

Received: 5 August 2008/Revised: 14 October 2008/Accepted: 9 December 2008/Published online: 28 December 2008

(C) The Author(s) 2008. This article is published with open access at Springerlink.com

\begin{abstract}
We sequenced five BAC clones of Brassica oleracea doubled haploid 'Early Big' broccoli containing major genes in the aliphatic glucosinolate pathway, and comparatively analyzed them with similar sequences in $A$. thaliana and B. rapa. Additionally, we included in the analysis published sequences from three other $B$. oleracea BAC clones and a contig of this species corresponding to segments in A. thaliana chromosomes IV and V. A total of $2,946 \mathrm{~kb}$ of B. oleracea, 1,069 $\mathrm{kb}$ of $B$. rapa sequence and $2,607 \mathrm{~kb}$ of $A$. thaliana sequence were compared and analyzed. We found conserved collinearity for gene order and content restricted to specific chromosomal segments, but breaks in collinearity were frequent resulting in gene absence likely not due to gene loss but rearrangements. $B$. oleracea has the lowest gene density of the three species, followed by B. rapa. The genome expansion of the Brassica species, B. oleracea in particular, is due to larger introns and gene spacers resulting from frequent insertion
\end{abstract}

Communicated by R. Schmidt.

Electronic supplementary material The online version of this article (doi:10.1007/s00299-008-0661-3) contains supplementary material, which is available to authorized users.

D. Qiu · M. Gao · C. Quiros $(\bowtie)$

Department of Plant Sciences, University of California,

Davis, CA 95616, USA

e-mail: cfquiros@ucdavis.edu

Present Address:

M. Gao

Department of Agronomy, University of Kentucky,

Lexington, KY 40546, USA

G. Li

Department of Plant Science, University of Manitoba, Winnipeg, MB R3T2N2, Canada of DNA transposons and retrotransposons. These findings are discussed in relation to the possible origin and evolution of the Brassica genomes.

Keywords Synteny - Gene mapping · Glucosinolates . Comparative genomics $\cdot$ Transposable elements

\section{Introduction}

Genome analyses in the model species Arabidopsis thali$a n a$, is a useful tool for comparative genomic studies in the related Brassica genus which include important crop species. Comparative mapping between the genomes of crop plants and their respective model species is becoming a common approach for the identification of markers and candidate genes for mapping studies and to expedite positional gene cloning. Genome sequencing projects for $B$. rapa and B. oleracea are in the process, providing an opportunity to analyze and study the genome changes associated with the origin and evolution of these species in relation to A. thaliana (Ayele et al. 2005; Lim et al. 2006; Yang et al. 2006; Hong et al. 2006).

The genus Brassica includes three main cultivated species, B. nigra, $n=8 ; B$. oleracea, $n=9$; and $B$. rapa, $n=10$, all of which function genetically as diploids. However, early evidence (Sikka 1940), indicated that these species are paleopolyploids, which is also the case for A. thaliana (Blanc et al. 2000). It is widely accepted that Brassica species and A. thaliana are diverged from a common ancestor of about 14.5-20.4 million years ago (Yang et al. 1999). The current thinking is that the common progenitor of the Brassicaceae had a basic genome of $n=4$ chromosomes, which underwent a whole genome duplication 24-40 Mya producing a tetraploid species of 
$2 n=4 x=16$ (Henry et al. 2006). The genome of this putative species was similar to the present genomes of A. lyrata and Capsella rubella $(2 n=16, \sim 230 \mathrm{Mbp}$ of DNA), from which presumable the genomes of the Brassica species derive (Schranz et al. 2006). A. thaliana evolved from this common ancestral species 4-5 Mya after it became diplodized and suffered general gene loss and chromosomal rearrangements including fusions or fissions resulting in the present genome of $n=5$ and $157 \mathrm{Mb}$ of DNA. (Johnston et al. 2005; Henry et al. 2006). Based on samplings of less than $2 \%$ of the genome, either by molecular marker map construction (Lagercrantz 1998; O'Neill and Bancroft 2000; Park et al. 2005; Parkin et al. 2005; Rana et al. 2004; Schmidt et al. 2003) and FISH (Lysak et al. 2005; Ziolkowski et al. 2006), it has been proposed that the Brassica diploid species are also evolved from the $2 n=4 x=16$ ancestral species after additional rounds of genome duplication, resulting in an hexaploid ancestor. This would explain in part the increase in DNA content from $230 \mathrm{Mbp}$ to 529-696 Mbp (Johnston et al. 2005) reported for monogenomic cultivated Brassica species. However, it ignores the fact that ploidy changes are changes in chromosome number. The monogenomic Brassica species have already identical or similar chromosome numbers than those in the putative ancestral species $(n=8$, 9 and 10). It also ignores the role of transposable elements which has been estimated to expand as much as $20 \%$ of the B. oleracea genome (Zhang and Wessler 2004). Little is known about the genomic structure of the Brassica diploid species. The two main cultivated species $B$. oleracea and $B$. rapa have diverged $7.3 \pm 4$ Mya (Wroblewski et al. 2000) and there is high synteny conservation for at least half of the chromosomes (Parkin et al. 2005). In the present study, we analyze similar sequences which harbor major glucosinolate genes in B. oleracea, B. rapa and A. thaliana in an effort to provide additional clues on the structure and evolution of Brassica genome.

\section{Materials and methods}

\section{Brassica oleracea $\mathrm{BAC}$ sequencing}

BAC clones B47M9, B67C16, B77C13, B59J16 and B16J1 originate from B. oleracea var. italica (broccoli) doubled haploid 'Early Big' library (Gao et al. 2004). These clones were selected because they harbor major genes in the aliphatic glucosinolate pathway. Clones B47M9, B67C16, B77C13 and B16J1 were outsourced for sequencing 454 Life Sciences (Bradford CT) using pyrosequencing. B59J16 was sequenced at the CA\&ES Genomics Facility (CGF) in the UCD campus following traditional techniques (Gao et al. 2004). Gaps were filled by a combination of primer walking and shotgun sequencing of sub-clones at both the sides of gaps. The summary of assemble details is shown in Supplementary Table 1. Final error rate was estimated using CONSED which is less than $1 \mathrm{bp}$ per $100 \mathrm{~kb}$. These sequences were deposited in GenBank under the following accession numbers: B16J1 (EU579454), B67C16 (EU581950), B77C13 (EU579455), B47M9 (EU673963) and B59J16 (EU568372). For comparison, we added to the analysis, sequences of three other BACs of the same broccoli variety, B21H13 (Gao et al. 2004), B19N3 (Gao et al. 2005) and B21F5 (Gao et al. 2006). Additionally, we included $B$. oleracea contigs in the analysis sequenced by Town et al. (2006) by comparing them to their corresponding $B$. rapa sequences in the public domain: BAC clones KBrH015M19 (AC172876), KBrB077F22 (AC189466), KBrB063K02 (AC189420), KBrH1070K21 (DQ369749), KBrH093K03 (AC155347), KBrB021P11 (AC189261), KBrS005L11 (AC189638), KBrH077A05 (AC155343) and KBrB080C12 (AC189471). The same method was used to analyze the sequences of these BACs.

Sequence analysis and gene-prediction

The BAC sequence was analyzed for protein-coding genes with the following gene-prediction of A. thaliana software: GenScan (Burge and Karlin 1997) and TwinScan, by comparing conserved regions in the DNA of both species (Flicek et al. 2003). The sequence of BACs was aligned with its corresponding A. thaliana sequences with BLAST 2.2.9 (Altschul et al. 1997). The BAC sequence was also compared to Arabidopsis, Brassica, and Oryza sativa ESTs, cDNAs, and CDS using BLAST and FASTA with NCBI, AGI and TIGR database (www.tigr.org/tdb/e2k1/bog1/) to analyze gene conservation. The conserved regions were translated into protein and tBLASTn applied to the GenBank protein database to adjust exon-intron boundaries (05/01/2008). The transposable elements (TE) in the sequences were predicted and located with the program "RepeatMasker" (A.F.A. Smith and P. Green, unpublished data, http://www.repeatmasker. org/) and BLASTN and BLASTX searches to the GenBank database to find by comparison all types of reported transposable elements (05/01/2008). The 'bases masked' number is calculated from the total number of basepair masked sequences. The 'bases masked' include the retroelements, DNA Transposons, low-complexity DNA and simple repeats.

\section{Results}

Annotation of the five B. oleracea BACs harboring GSL genes

The five B. oleracea BACs B67C16, B47M9, B77C13, B59J16 and B16J1 sequenced were selected because they 
harbor a major aliphatic glucosinolate (GSL) gene $B o G S$ OH (At2g25450), BoCS-lyase (At2g20610), BoCYP79F1 (At1g16410), BoGSL-PROb (At1g18500) and BoS-GT (At1g24100), respectively. The annotation of the genes in these BACs is shown in Supplementary Tables 2, 3, 4, 5 and 6 . The other three BAC clones previously sequenced and analyzed include $\mathrm{B} 21 \mathrm{H} 13$ harboring $B o G S L-A L K a$ and $B o G S L-A L K b, \mathrm{~B} 19 \mathrm{~N} 3$ harboring BoGSL-ELONG and $B o G S L-E L O G-L$ and B21F5 harboring BoGSL-PRO (Gao et al. 2004, 2005, 2006).

Characteristics of $B$. oleracea and $B$. rapa sequences in relation to $A$. thaliana

A total of $2,946 \mathrm{~kb}$ of $B$. oleracea sequence, including all eight broccoli BAC clones and the contigs published by Town et al. (2006), 1,069 $\mathrm{kb}$ of $B$. rapa sequence and $2,607 \mathrm{~kb}$ of corresponding $A$. thaliana sequence were compared and analyzed. Most of the comparative data could be generated between $B$. oleracea and A. thaliana, since corresponding $B$. rapa sequences were available only for half of the B. oleracea clones, B16J1, B21F5 B67C16. Table 1 shows a global summary of the features of these sequences for all three species. All the genes in the sequences of the three species were taken into account, however, only $48 \%$ of the B. oleracea and $71 \%$ of the $B$. rapa genes had counterparts in their corresponding A. thaliana sequences due to breaks in synteny. BLAST search in the $B$. oleracea and $B$. rapa databases for the absent genes indicated that at least $50 \%$ had homologs somewhere else in the genome (data not shown). The global gene comparison for the chromosomal segments compared revealed that on an average $B$. rapa genes were significantly longer $(3,218 \mathrm{bp})$ than those in $B$. oleracea $(2,721 \mathrm{bp})$ and the latter tended to be longer than in A. thaliana $(2,310 \mathrm{bp})$, but the difference was not statistically significant. These differences were associated mostly to intron size, which was significantly larger in $B$. rapa compared to the other two species. Gene density was lowest for B. oleracea (one per $8.0 \mathrm{~kb}$ ) followed by $B$. rapa (5.4 kb) and A. thaliana (one per $4.5 \mathrm{~kb}$ ). This parameter was associated to gene spacer size which was larger in the Brassica species, B. oleracea in particular (Table 1). Taking into account only the genes conserving collinearity, gene size as well as exon size and number, were the same for all three species. However, intron size was different between $A$. thaliana and the Brassica species, but not between $B$. olerace $a$ and $B$. rapa.

Annotation of the eight $B$. oleracea BAC sequence $(745.8 \mathrm{~kb})$ resulted in the construction of a total of 94 gene models (Table 2). These include the updated annotation of BACs B19N3, B21F5 and B21H13 previously reported (Gao et al. 2004, 2005, 2006). Considering all the eight clones, we could classify them by gene density. B21H13 has the highest density with 23 gene models in $101.5 \mathrm{~kb}$ and B47M9 has the lowest density with eight gene models in $104.6 \mathrm{~kb}$ (Table 2). A total of 89 gene models were annotated in $495.7 \mathrm{~kb}$ sequence of four $B$. rapa $\mathrm{BAC}$ clones that could be partially aligned to the four $B$. oleracea BAC clones listed at the beginning of this section (Table 2; Fig. 1). The B. rapa BACs had higher gene density than the $B$. oleracea BACs, in agreement with the global sequence comparison summarized in Table 1. In order to get a better picture of the alignment of the
Table 1 Features of eight $B$. oleracea and four B. rapa $\mathrm{BAC}$ clones

* $P<0.05 ; * * P<0.01$

\begin{tabular}{llll}
\hline & $\begin{array}{l}\text { B. oleracea } \\
\text { BAC clone }\end{array}$ & $\begin{array}{l}\text { B. rapa } \\
\text { BAC clone }\end{array}$ & Arabidopsis \\
\hline Sequence length & $745.8 \mathrm{~kb}$ & $495 \mathrm{~kb}$ & $449 \mathrm{~kb}$ \\
$\mathrm{G}+$ C content overall (\%) & 36.35 & 36.81 & 35.8 \\
Protein-coding DNA (\%) & 45.40 & 43.68 & 42.90 \\
Non-coding region (\%) & 33 & 33.80 & 32.50 \\
Total number of genes & 94 & 89 & 213 \\
Average gene size (bp) & $2721^{*}$ & 3,218 & $2,310^{*}$ \\
Average gene density (bp per gene) & $8012^{*}$ & 5,436 & 4,450 \\
Average number exons per gene & 5.6 & 6.9 & 5.3 \\
Average exon size (bp) & $225^{* *}$ & 272 & $258^{*}$ \\
Average number introns per gene & 4.6 & 5.9 & 4.3 \\
Average intron size (bp) & $206^{*}$ & $240^{* *}$ & $153^{* *}$ \\
Average spacer size (bp) & $6,368^{* *}$ & 3,369 & 2,470 \\
Average gene size (bp) for homologus gene & 2,569 & 2,685 & 2,436 \\
Average exon size (bp) for homologus gene & 235 & 254 & 246 \\
Average intron size (bp) for homologus gene & 225 & 238 & $176^{*}$ \\
\hline
\end{tabular}




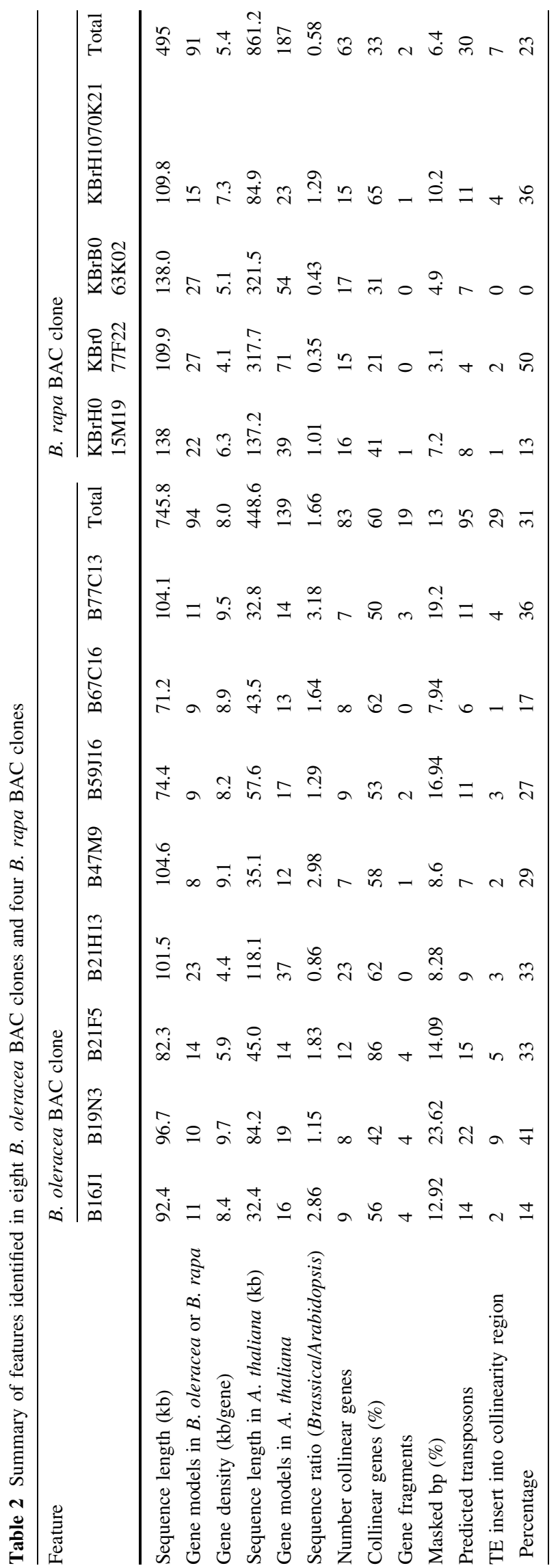

Fig. 1 The comparison map of B. oleracea and Brassica rapa BAC clones with $A$. thaliana. Open right arrow DNA transposons, filled right arrow retroelements, open rectangle gene fragments. Vertical lines indicates sequence contigs. The triangle by each gene model name indicates the coding strand of the gene

corresponding sequences of A. thaliana and B. rapa, the BAC clones of the latter species were aligned to the physical map of $A$. thaliana (Fig. 1).

Annotation of B. oleracea contigs A B, C, D and G, covering a total of $1,518 \mathrm{~Kb}$, resulted in the construction of 378 gene models (Table 3). Five B. rapa BAC clones could be partly aligned with these five $B$. oleracea contigs. A total of 60 gene models were annotated in $574 \mathrm{~kb}$ sequence of these corresponding B. rapa BAC clones (Table 3). Contrary to the trend found for the B. oleracea BAC clones, higher gene density were observed in the contigs for this species $(4.0 \mathrm{~kb})$ than in A. thaliana $(4.6 \mathrm{~kb})$ and $B$. rapa $(5.6 \mathrm{~kb})$.

All of these Brassica contigs have a high level of DNA sequence conservation with their counterparts in A. thaliana. One hundred and thirty nine and 187 A. thaliana gene models were identified in the corresponding region of eight $B$. oleracea and four B. rapa BAC clones, respectively (Fig. 1). Two hundred and thirteen and 136 A. thaliana gene models were identified in the corresponding region of five $B$. oleracea contigs and another five $B$. rapa BAC clones, respectively (Fig. 1).

DNA sequence conservation and collinearity between Brassica and Arabidopsis

In general, collinearity in the sense of finding corresponding genes in the same order and orientation was high among all the three species. In the eight $B$. oleracea BACs, $88 \%$ of the genes (83 of 94) conserved order with $139 A$. thaliana genes in their corresponding regions (Table 2; Fig. 1). Sixty nine percent of B. rapa genes (63 of 91) conserved order with 187 A. thaliana genes in their corresponding regions (Table 2; Fig. 1). However, gene content was often different among all the three species in corresponding segments, due to frequent interspersed gene absence in the Brassica species in relation to Arabidopsis (Figs. 1, 2). Thus, when one considers in the comparison all the genes in the corresponding segments, the collinearity drops significantly to $60 \%$ between $B$. oleracea and A. thaliana and $33 \%$ between B. rapa and A. thaliana (Table 2). These values were higher for the five B. oleracea contigs, $70 \%(144 / 213)$ of the genes conserved collinearity to A. thaliana and 52\% (73/136) for their corresponding B. rapa BAC sequences (Table 3; Fig. 2).

In a few instances genes present in chromosomal segments of Brassica species were absent in corresponding segments of $A$. thaliana. For example comparing 


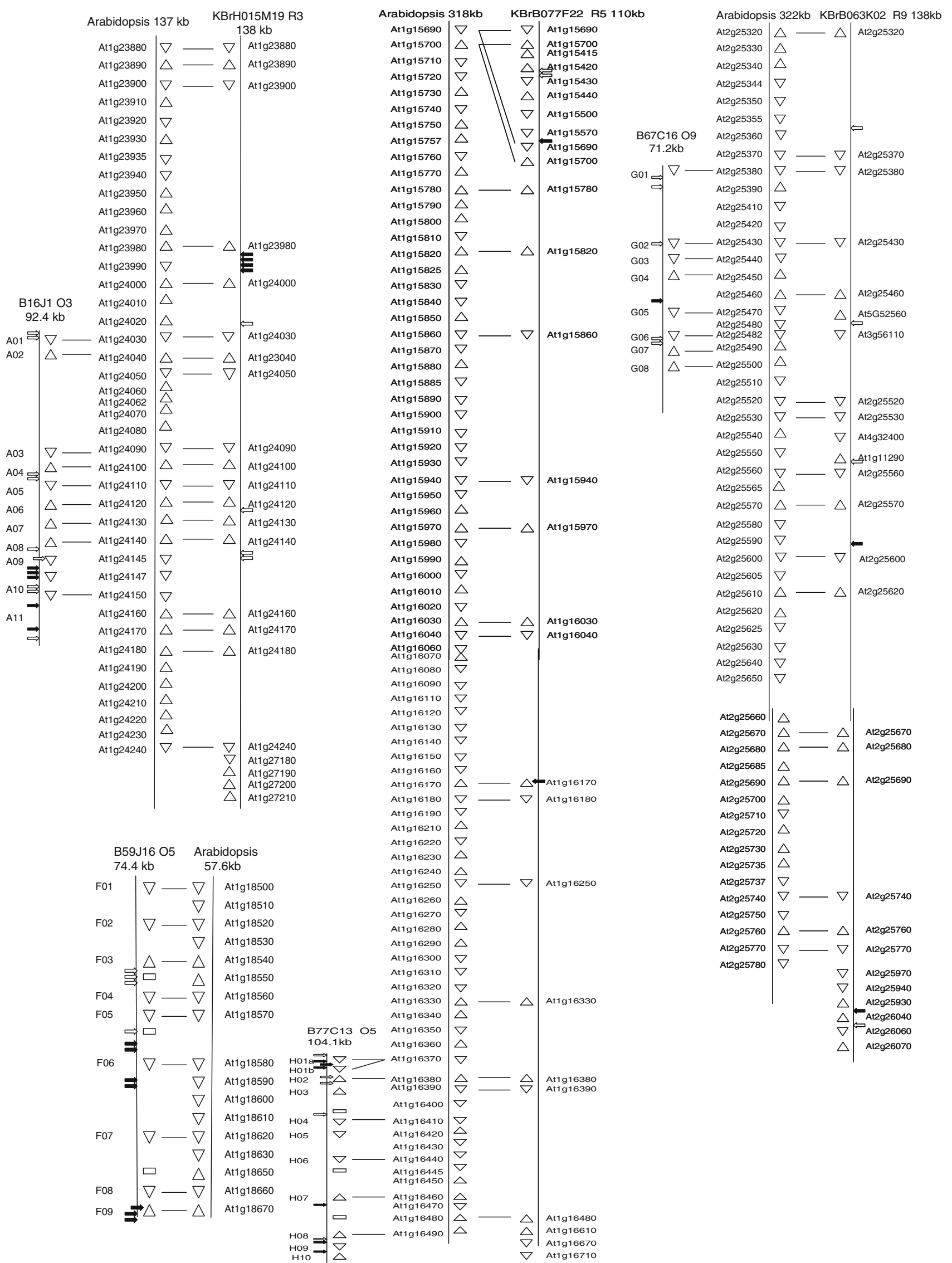




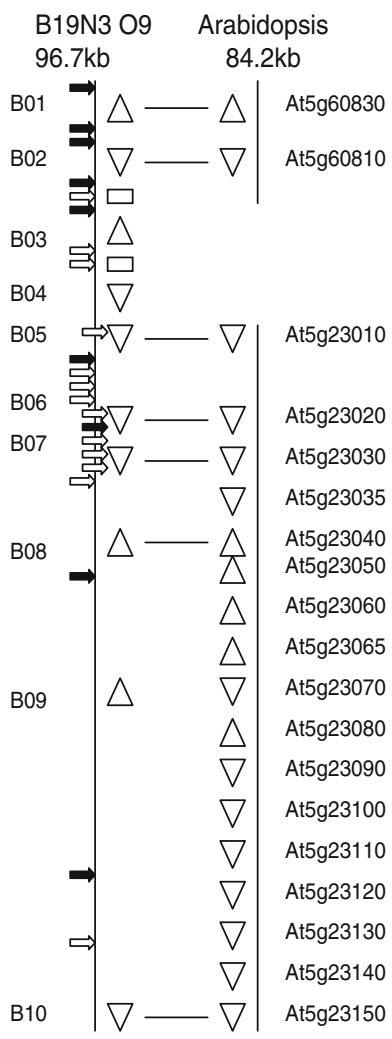

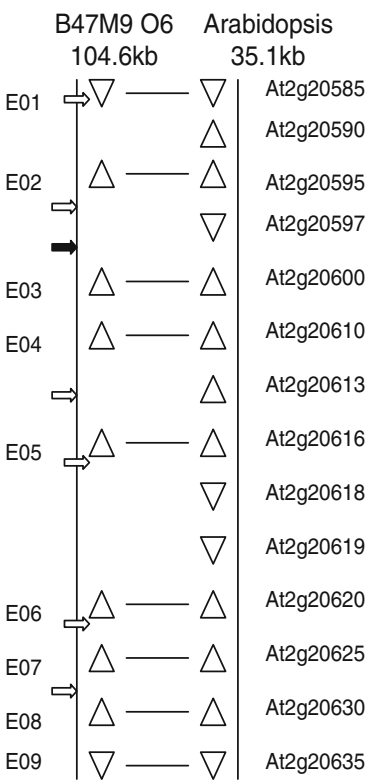

\section{Arabidopsis $\quad \mathrm{KBrH} 1070 \mathrm{~K} 21 \mathrm{R} 5 \quad \mathrm{~B} 21 \mathrm{H} 13 \mathrm{O}$ Arabidopsis $\begin{array}{llll}85 \mathrm{~kb} & 110 \mathrm{~kb} & 101.5 \mathrm{~kb} & 118.1 \mathrm{~kb}\end{array}$}

At1g18190 $|\triangle \longrightarrow \triangle|$ At1g18190 At1g18200 $\triangle$

At1g18210 $\triangle \longrightarrow \triangle$ At1g18210 At1g18220 At1918250 At1g18260 At1g18265 At1g18270 At1918280 At1g18290 At1g18300 At1918310 At1g18330 At1918335 At1g18340 At1g18350 At1g18360 At1918370 At1918380

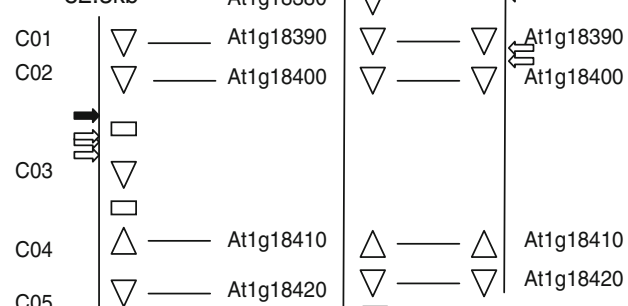
$\mathrm{C} 06$

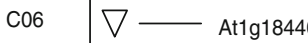

$\nabla 07 \quad \nabla-{ }_{\text {At1g18460 }}^{\text {At1g18440 }}$ $\triangle \longrightarrow \triangle$ At1g18250 $\triangle \longrightarrow \triangle$ At1g18265 $_{\triangle}$ $\triangle-\triangle$ At1g18270 $\nabla-\nabla$ At1g18280

$\nabla-\nabla$ At1g18290

$\nabla-\nabla$ At1g18300<smiles>C#[Al]</smiles>

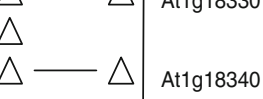

$\nabla$

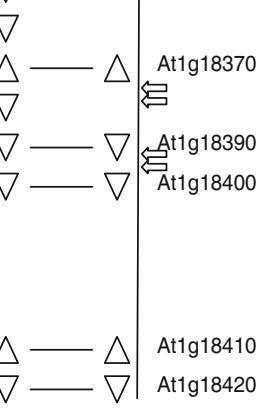

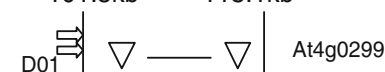

D02 $\nabla-\nabla \quad$ At4g03000

D03 $\nabla-\nabla \quad$ At4g03010

D04 $\triangle-\triangle \quad$ At4g03020

D05 $\triangle-\triangle \quad$ At4g03030

\begin{tabular}{ll|l}
$\nabla$ & At4g03040 \\
$\nabla$ & At4g03050
\end{tabular}

D06b $\triangle \longrightarrow \triangle \quad$ At4g03060

D07a $\nabla \longrightarrow \nabla \quad$ At4g03070

D07b $\nabla / \triangle$ At4g03080

D08 $\Rightarrow \triangle-\triangle \quad$ At4g03090

D09 $\nabla-\nabla \quad$ At4g03100

D10 $\triangle-\triangle \quad$ At4g03110

D11 $\triangle \triangle \triangle$ At4g03115

D12 $\nabla-\nabla \quad$ At4g03120

D13 $\triangle-\triangle \quad$ At4g03130

$\nabla$ At4g03135

\begin{tabular}{l|l|l} 
D14 & $\nabla-\nabla$ & At4g03140 \\
D15 & At4g03150
\end{tabular}

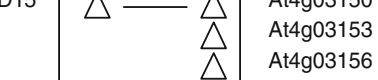

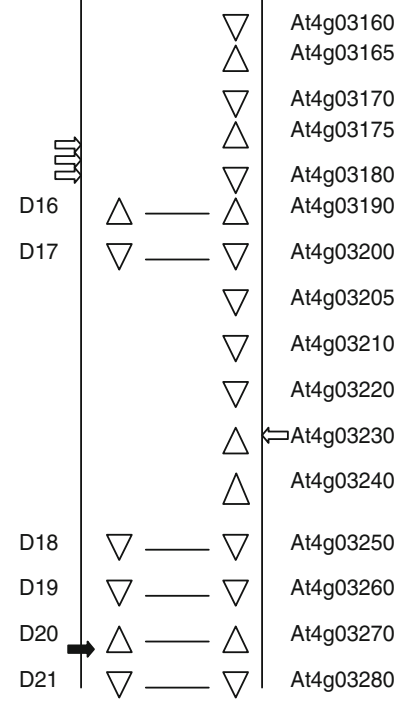

Fig. 1 continued

B. oleracea with A. thaliana, genes A09-A11 on B16J1, B09 on B19N3, C03 and C09 on B21F5, and H03, H05, $\mathrm{H} 09$ and $\mathrm{H} 10$ on $\mathrm{B} 77 \mathrm{C} 13$ are absent in A. thaliana.

Often the genes showing the syntenic changes were flanked by unrelated partial genes (Fig. 1). In fewer instances, the changes were associated to chromosomal rearrangements, such as is the case for genes B01-B03 in B19N3. In a few cases, tandem duplicates could be observed in B. oleracea, for example genes D06a and D06b, H01a and H01b. A similar situation was observed when $B$. rapa and $A$. thaliana were compared. Clone KBrB063K02 corresponding to chromosome II of $A$. thaliana had two 
Table 3 Summary of features identified in seven B. oleracea contigs and five B. rapa BAC clones

\begin{tabular}{|c|c|c|c|c|c|c|c|}
\hline \multirow[t]{2}{*}{ Feature } & \multicolumn{7}{|c|}{ B. rapa $\mathrm{BAC}$ clone } \\
\hline & $\begin{array}{l}\text { B. oleracea } \\
\text { contigs }^{\mathrm{a}}\end{array}$ & $\begin{array}{l}\text { KBrH0 } \\
93 \mathrm{~K} 03\end{array}$ & $\begin{array}{l}\mathrm{KBrB} 0 \\
21 \mathrm{P} 11\end{array}$ & $\begin{array}{l}\mathrm{KBrS0} \\
05 \mathrm{I} 11\end{array}$ & $\begin{array}{l}\mathrm{KBrB} 0 \\
80 \mathrm{C} 12\end{array}$ & KBrH077A05 & Total \\
\hline Sequence length $(\mathrm{kb})$ & 2200 & 78.3 & 116 & 127.7 & 138.3 & 113.3 & 574 \\
\hline Gene models in $B$. oleracea or B. rapa & 539 & 14 & 24 & 19 & 21 & 25 & 103 \\
\hline Gene density (kb/gene) & 6.6 & 5.6 & 4.8 & 6.7 & 6.6 & 4.5 & 5.6 \\
\hline Sequence length in $A$. thaliana $(\mathrm{kb})$ & 498 & 79.9 & 122.0 & 103.8 & 100.8 & 107.8 & 514 \\
\hline Gene models in $A$. thaliana & 271 & 28 & 36 & 30 & 25 & 33 & 136 \\
\hline Sequence ratio (BrassicalA. thaliana) & 1.70 & 0.98 & 0.95 & 1.23 & 1.37 & 1.05 & 1.12 \\
\hline Number of collinear genes & 177 & 9 & 19 & 15 & 14 & 16 & 73 \\
\hline Collinear genes $(\%)$ & 144 & 32 & 52 & 50 & 56 & 49 & 52 \\
\hline Gene fragments & $10 ?$ & 2 & 0 & 1 & 1 & 3 & 7 \\
\hline Masked bp (\%) & 18 & 18 & 7 & 16 & 13 & 13 & 13 \\
\hline Predicted transposons & 69 & 14 & 10 & 21 & 11 & 13 & 69 \\
\hline TE insert into collinearity region & 14 & 5 & 7 & 6 & 4 & 1 & 23 \\
\hline Percentage & 25 & 36 & 70 & 29 & 36 & 8 & 33 \\
\hline
\end{tabular}

${ }^{a}$ Data extracted from Town et al. (2006)

homologs inserted from chromosomes V and III, respectively and two from homologs on chromosomes IV and I, respectively (Fig. 1). Homologs corresponding to contiguous genes At1g15690 and At1g15700 were segmentally duplicated in $B$. rapa, a few genes downstream the original location next to a retroelement.

We could compare B. oleracea and B. rapa for segments corresponding to four sets of BAC clones. The general theme of missing genes in either B. rapa or B. oleracea could be observed as the main cause for collinearity disruptions. For $B$. oleracea BAC B16J1, there was almost perfect colinearity with $B$. rapa, spanning from the homologs of At1g24030-At1g24140, except for At1g24050 that is missing in B. oleracea. Four other homologs next to At1g24050, which includes At1g24060-At1g24080, were missing in both the Brassica species. Similar to A. thaliana, B. oleracea genes A09, A10 and A11, which are homologous to genes of At2g32430, At1g70140 and At1g67020, respectively, were missing in the corresponding segments of $B$. rapa (Fig. 1). Collinearity for the segment corresponding to B21F5 was almost identical in both species, except for the presence of gene $\mathrm{C} 03$, corresponding to homolog (At2g13865) and a partial gene in B. oleracea and absent in both $B$. rapa and A. thaliana (Fig. 1). For BAC clones $\mathrm{B} 67 \mathrm{C} 16$, it has higher collinearity with its corresponding sequence in A. thaliana than with that of $B$. rapa. Genes G03 and G04 were absent in B. rapa, whereas the homolog corresponding to gene At2g25460 was missing in $B$. oleracea, and the segment spanned by genes G05-G08 in B. oleracea was absent in B. rapa. At least four other segments present in A. thaliana were absent in B. rapa,
At2g25540-At2g25570, At2g25580 and At2g25590, At2g25605 and At2g25610, and At2g25625-At2g25670 (Fig. 1). No B. oleracea sequence was available to tell whether these segments were also absent in the corresponding segment for this species. The number of absent genes in $B$. rapa for the segment corresponding to $B$. oleracea $\mathrm{BAC}$ clone was very extensive. $B$. oleracea genes $\mathrm{H} 01, \mathrm{H} 03, \mathrm{H} 05 \mathrm{H} 09$ and H10 were absent in B. rapa (Fig. 1). Additionally, by comparing the corresponding segments between $B$. rapa and A. thaliana, two blocks of genes were missing in the former species, At1g16260 At1g16320 and At1g16340-At1g16370 (Fig. 1).

Transposable elements

We detected 95 TEs in the eight $B$. oleracea BACs corresponding to a masked base percentage of $13 \%$, whereas in B. rapa these numbers were much lower, 30 and $6.4 \%$, respectively (Table 4$)$. The TE density in the $B$. oleracea BACs was $0.13(95 / 746 \mathrm{~kb})$, whereas in the corresponding segments of $B$. rapa was $0.06(30 / 495 \mathrm{~kb})$.

Also, we detected 243 TEs in the five B. oleracea contigs corresponding to a masked base percentage of $18 \%$, whereas in B. rapa these numbers were much lower, 34 and $13 \%$, respectively (Table 5). The TE density in the $\mathrm{B} . \mathrm{ol}$ eracea contigs was $0.16(243 / 1518 \mathrm{~kb})$, whereas in the corresponding segments of $B$. rapa it was $0.12(69 / 574 \mathrm{~kb})$.

In the B. oleracea BACs the percentage of retroelements (class $1 \mathrm{TEs}$ ) is $61 \%$, and of DNA transposons (class 2 TEs) is $39 \%$. The opposite is true for B. rapa where the percentage of retroelments is 34 and $66 \%$ for DNA 
transposons. For the B. oleracea contigs, we found similar frequency of these types of elements, class 1 TEs is $65 \%$ and class 2 TEs is $35 \%$. However, for the corresponding $B$. rapa segments of the $B$. oleracea contigs, the percentage for class 1 and 2 TEs were nearly the same (51 and 49\%).

In the total B. oleracea sequence analyzed, there are 0.7 TEs per gene and $0.15 \mathrm{TE}$ per $1 \mathrm{~kb}$ of sequence, and 218 masked bases per $1 \mathrm{~kb}$ of sequence. In the $B$. rapa BACs there are 0.5 TEs per gene and 0.09 TEs per $1 \mathrm{~kb}$ of sequence, 96 masked bases per $1 \mathrm{~kb}$ of sequence. The number and masked base percentage of class 2 TEs is more than the class 1 TEs in both Brassica species (Table 6). Sixteen percent of $B$. oleracea sequence, $10 \%$ of $B$. rapa sequence and $4 \%$ of $A$. thaliana sequence corresponds to TE.

The LTR elements were the main type in the retroelements in both Brassica species. The En-spm type is predominant in the DNA transposons of $B$. oleracea and the hAT type is predominant in B. rapa.

In the eight $B$. oleracea $\mathrm{BACs}, 17$ transposable elements, 13 DNA transposons and four retroelements were inserted into genes. Only one retroelement inserted into a B. rapa gene, which is the ortholog for At1g16170 (Fig. 1).

The insertion of TEs was not frequently associated to chromosomal segments displaying breaks in synteny among species. Thirty one percent of TEs in eight $\mathrm{B}$. oleracea BACs and $49 \%$ in the contigs were inserted into regions maintaining collinearity with $A$. thaliana, whereas $23 \%(7 / 30)$ of TEs were inserted into regions maintaining collinearity in four $B$. rapa clones with $A$. thaliana (Table 2; Fig. 1).

Little conservation of transposable elements insertions was observed among the three species. Only one SINE type TE with same sequence was found in the corresponding location in $B$. oleracea contig $\mathrm{B}$ and $B$. rapa $\mathrm{BAC}$ KBRH093K03. This TE is of the AtSB6 type and has 68 bp.

\section{Discussion}

Differential gene content for corresponding segments in the three species

Most of the comparative genomics work done to date among Brassica species with reference to A. thaliana, are based on physical and genetic mapping procedures (Rana et al. 2004; Parkin et al. 2005; Park et al. 2005). Comparative sequencing of specific chromosomal regions provides useful new information on gene density, synteny and conservation of gene collinearity along these segments (Gao et al. 2004, 2005, 2006; Yang et al. 2006; Town et al. 2006). In general, gene density for the chromosomal
Fig. 2 The comparison map of five B. oleracea contigs and five Brassica rapa BACs clones with A. thaliana

segments studied was highest for A. thaliana, followed by $B$. rapa and $B$. oleracea. The limited sequencing data of $B$. rapa available for this study did not allow us to ascertain orthology with B. oleracea BAC clones B47M9, B67C16, B77C13, B59J16 and B16J1. However, the conclusion that can be reached from our survey is that for the chromosomal segments studied, gene density is lower in the Brassica species than in A. thaliana. In terms of genome expansion, Brassica/Arabidopsis sequence length ratios for the $B$. oleracea sequence analyzed is 1.7 , ranging from 0.86 to 3.2 (Tables 2, 3). However, gene density is not uniform across the genome where regions of higher and lower gene density might co-exist, such as is the case for the region covered by BAC clone B21H13. A similar situation is observed for $B$. rapa, although gene density in this case is higher than in $B$. oleracea (Tables 2, 3). Lower density in the Brassica species is associated to larger introns and spacers and to extensive gene rearrangement resulting in the absence of genes in otherwise collinear chromosomal segments with A. thaliana. The fact that approximately $50 \%$ of the genes absent in the compared segments which can be accounted for the Brassica data bases (which are incomplete), indicates that most of these genes have not been lost and are somewhere else in the genomes of B. rapa and B. oleracea. Due to these rearrangements, the breaks in synteny between Brassica and A. thaliana can be quite extensive, depending on the chromosomal segment compared. This is also true between the two Brassica species, which evolutionarily are considered to be in the same lineage (Warwick and Black 1991). This is in agreement with the results of Parkin et al. (2005) who estimated 74 gross rearrangements taking place between the $\mathrm{A}$ and $\mathrm{C}$ genome chromosomes of B. napus. Using RFLP markers, they were able to identify 21 conserved regions of $A$. thaliana duplicated and rearranged in the $\mathrm{A}$ and $\mathrm{C}$ genome chromosomes of $B$. napus. These conserved segments were on an average $9 \mathrm{Mbp}$ in length. Physical mapping studies by Park et al. (2005) comparing specific chromosomal segments for all three species; report also breaks in synteny mostly due to gene absence. The earlier report of Kowalski et al. (1994) had already suggested that conservation of synteny and gene content between A. thaliana and Brassica was limited to specific segments or genomic islands. However, as additional comparative sequencing data are accumulated, it is evident that these conserved islands are small. Furthermore, in spite of their phylogenetic close proximity, the genomes of $B$. oleracea and $B$. rapa have also undergone extensive structural changes resulting in segmental conservation of collinearity. Most of the changes observed are species specific, including gene duplications. Thus, these 

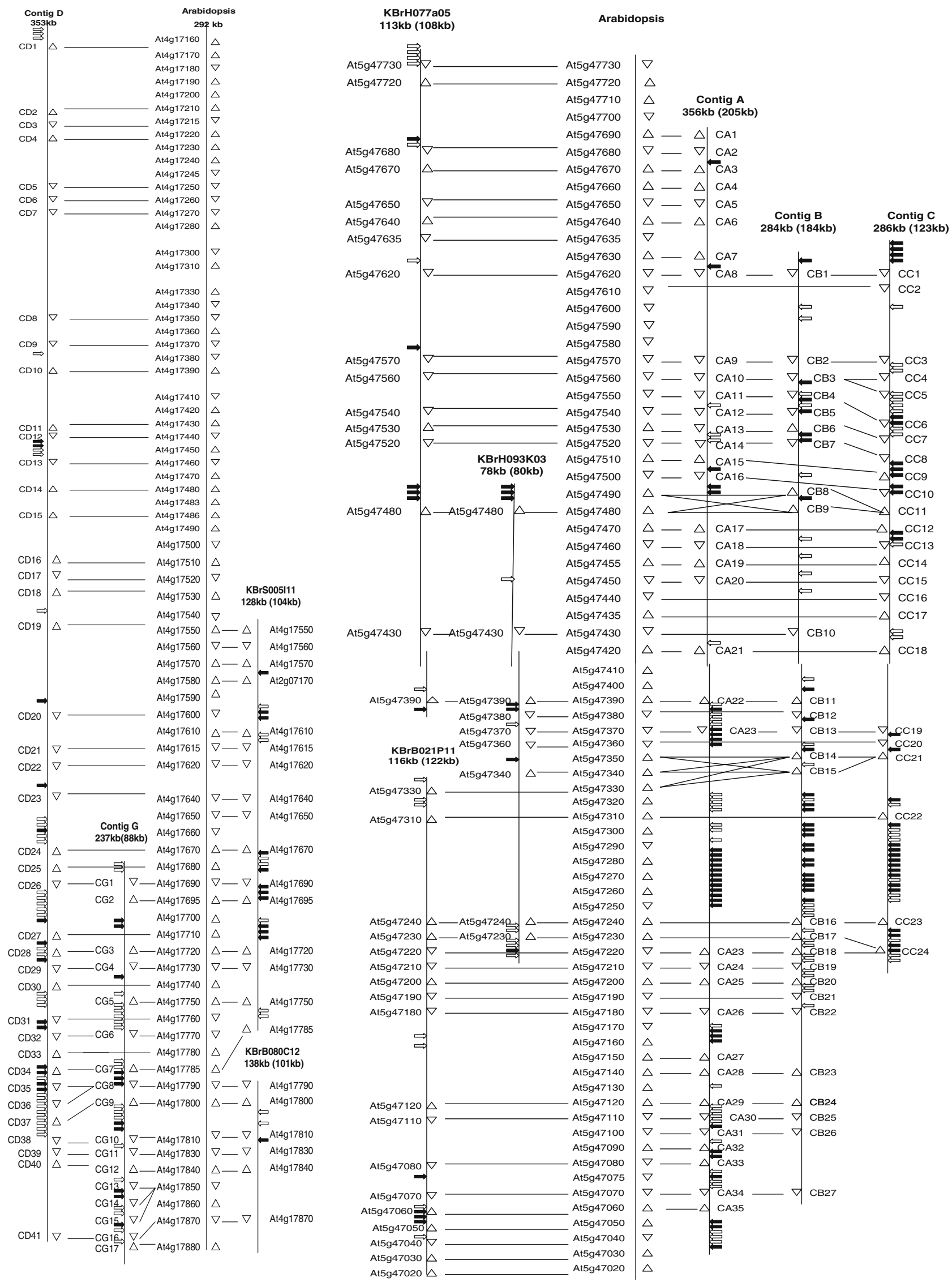


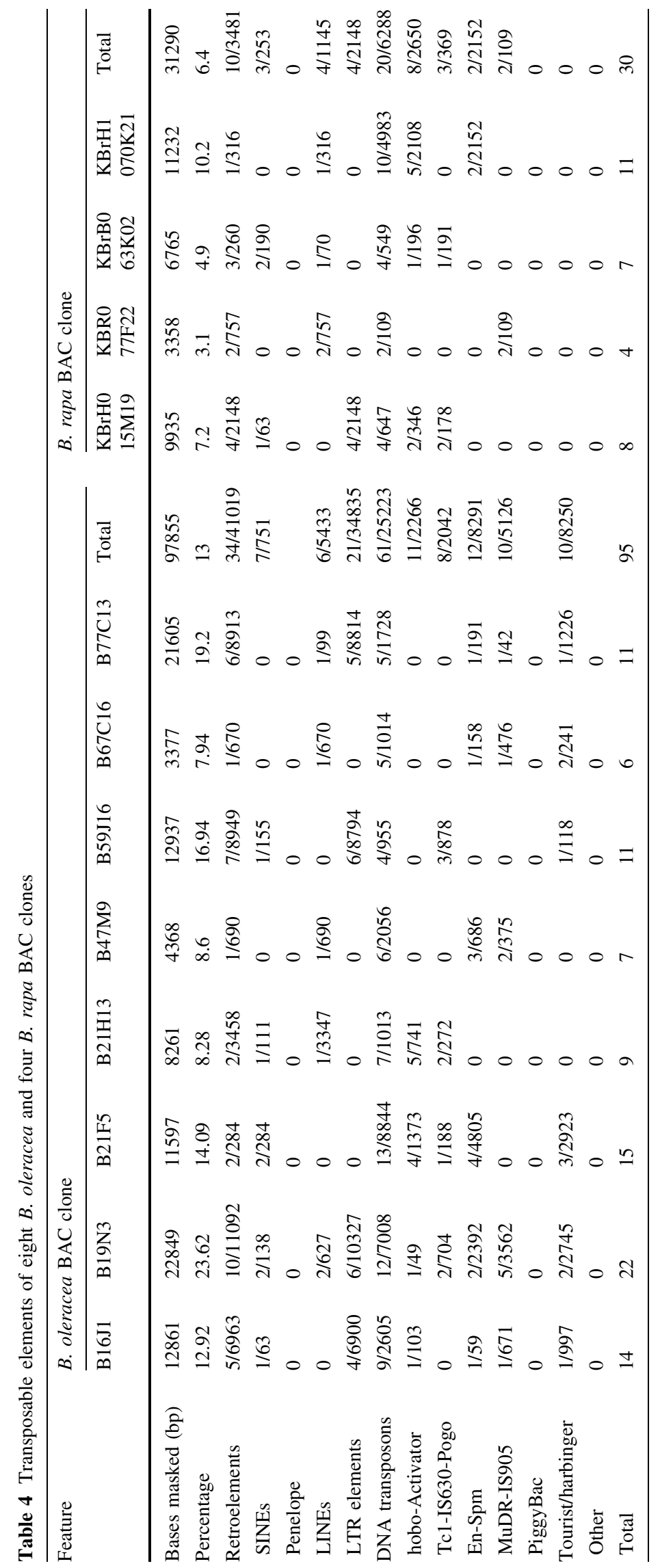


Table 5 Transposable elements of five B. oleracea contigs and five B. rapa BAC clones

\begin{tabular}{|c|c|c|c|c|c|c|c|}
\hline \multirow[t]{2}{*}{ Feature } & \multicolumn{2}{|c|}{ B. oleracea contigs } & \multicolumn{5}{|c|}{ B.rapa BAC clone } \\
\hline & Total & KBrH0 93K03 & KBrB0 21P11 & KBrS0 05I11 & $\mathrm{KBrB} 080 \mathrm{C} 12$ & KBrH077A05 & Total \\
\hline Bases masked (bp) & 397813 & 14027 & 7251 & 20673 & 18391 & 14633 & 74975 \\
\hline Pecentage & 18 & 18 & 7 & 16 & 13 & 12.9 & 13 \\
\hline Retroelements & $114 / 147617$ & $6 / 6029$ & $4 / 848$ & $14 / 14646$ & $4 / 1004$ & $6 / 8919$ & $34 / 31446$ \\
\hline SINEs & $6 / 606$ & $1 / 71$ & $1 / 135$ & $2 / 321$ & $2 / 282$ & $2 / 133$ & $6 / 942$ \\
\hline Penelope & $0 / 0$ & $0 / 0$ & $0 / 0$ & $0 / 0$ & $0 / 0$ & 0 & $0 / 0$ \\
\hline LINEs & $29 / 33842$ & $1 / 806$ & $3 / 713$ & $2 / 737$ & $2 / 722$ & $1 / 3933$ & $7 / 6911$ \\
\hline LTR elements & $79 / 113169$ & $4 / 5152$ & $0 / 0$ & $10 / 13588$ & $0 / 0$ & $3 / 4853$ & $17 / 23593$ \\
\hline DNA transposons & $129 / 79245$ & $8 / 2160$ & $6 / 2153$ & $7 / 10151$ & $7 / 7505$ & $7 / 1734$ & $35 / 23703$ \\
\hline hobo-Activator & $37 / 16174$ & $3 / 1214$ & $0 / 0$ & $2 / 307$ & $1 / 461$ & $5 / 1251$ & $10 / 3233$ \\
\hline Tc1-IS630-Pogo & $10 / 2332$ & $3 / 635$ & $5 / 924$ & $4 / 725$ & $2 / 414$ & $2 / 1519$ & $14 / 4217$ \\
\hline En-Spm & $20 / 43888$ & $0 / 0$ & $0 / 0$ & $0 / 0$ & $2 / 6185$ & $0 / 0$ & $0 / 6185$ \\
\hline MuDR-IS905 & $17 / 7254$ & $0 / 0$ & $0 / 0$ & $0 / 0$ & $0 / 0$ & $0 / 0$ & $0 / 0$ \\
\hline PiggyBac & $1 / 1310$ & $0 / 0$ & $0 / 0$ & $0 / 0$ & $0 / 0$ & $0 / 0$ & $0 / 0$ \\
\hline Tourist/harbinger & $4 / 5666$ & $0 / 0$ & $1 / 1205$ & $0 / 0$ & $0 / 0$ & $0 / 0$ & $1 / 1205$ \\
\hline Total & 243 & 14 & 10 & 21 & 11 & 13 & $34 / 31446$ \\
\hline
\end{tabular}

Table 6 Distribution of transposable elements in Brassica

\begin{tabular}{|c|c|c|c|c|c|c|}
\hline \multirow[t]{2}{*}{ Item } & \multicolumn{2}{|c|}{ Total TE (num/bp) } & \multicolumn{2}{|l|}{ Retroelments } & \multicolumn{2}{|c|}{ DNA transposons } \\
\hline & B. oleracea & B. rapa & B. oleracea & B. rapa & B. oleracea & B. rapa \\
\hline Total TE content & $338 / 495668$ & 99/91632 & $148 / 188636$ & $44 / 26008$ & $190 / 104468$ & $55 / 28257$ \\
\hline TE per Brassica gene & $0.7 / 1043$ & $0.5 / 569$ & $0.3 / 397$ & $0.2 / 162$ & $0.6 / 219$ & $0.5 / 175$ \\
\hline TE per $k b$ & $0.15 / 218$ & $0.09 / 96$ & $0.07 / 83$ & $0.04 / 27$ & $0.08 / 46$ & $0.04 / 29$ \\
\hline
\end{tabular}

changes have taken place after the separation of the oleracea and rapa lineages.

Differential TE content in B. oleracea and B. rapa

For the segments analyzed, we found a lower frequency of TEs in B. rapa than B. oleracea, which is in agreement with the smaller genome size of the former species. We estimated that for these segments, approximately $16 \%$ of $B$. oleracea sequence and $10 \%$ of $B$. rapa sequence consist of TEs, which is not far from a global TE estimate of $20 \%$ for B. oleracea by Zhang and Wessler (2004) and Katari et al. (2005). Of these, approximately $14 \%$ correspond to retroelements and 6\% to DNA transposons. For B. rapa, based on $60 \mathrm{Mb} \mathrm{BAC}$ end sequences, $12.3 \%$ of the sequences consist of TE sequences, of which $84 \%$ are retroelements and $11.4 \%$ are DNA transposons (Lim et al. 2006). Our estimate is also in agreement with this report. Considering that the TE content in A. thaliana is only $4 \%$ (Zhang and Wessler 2004) the accumulation of these elements has taken place after the separation of the Arabidopsis and Brassica lineages. Alix and Heslop-Harrison (2004) have analysed the diversity of retroelements in diploid and allotetraploid
Brassica species, where there is a distinct clustering of Copia-like retroelements in $\mathrm{C}$ genome much more than the $\mathrm{A}$ and $\mathrm{B}$ genome. This result is in agreement with our observation, $83 \mathrm{bp}$ retroelements in $1 \mathrm{~kb} B$. oleracea sequence is more than the $27 \mathrm{bp}$ in $B$. rapa.

When comparing TE insertions between $B$. oleracea and B. rapa, we found little conservation of TE elements. Only one insertion was shared between the two species. Therefore, it is evident that each species have followed their own path of TE acquisition, where the rate of accumulation of these elements has been higher in B. oleracea than in $B$. rapa.

Based on previous reports and our work, TE elements are ubiquitous in Brassica species and have an important role in genome evolution. Most likely current estimates for these elements will increase as progress is made on sequence annotation. For example, Lim et al. (2007) after analyzing close to 88,000 BAC clones have found that retrotransposons are major components of centromeres and peri-centromeric regions in most Brassica species. One can estimate the contribution of these elements as part of the Brassica genomes as follows: B. oleracea has a DNA content of $696 \mathrm{Mbp}$, of which 20\% (139 Mbp) are TEs. 
Assuming an even distribution of DNA in all nine chromosomes, each has then approximately $77 \mathrm{Mbp}$ of DNA. Thus, the increase in DNA by TEs in $B$. oleracea is equivalent to adding two chromosomes to its genome. If the ancestral Brassica lineage had $2 n=4 x=16$ (Henry et al. 2006), we will have to add another eight chromosomes to produce an hexaploid (Lysak et al. 2005; Parkin et al. 2005; Ziolkowski et al. 2006; Yang et al. 2006), which presumable would then have $2 n=6 x=24$. Therefore, a simpler explanation is to assume that the Brassica lineage diverged from the tetraploid ancestral lineage proposed by Henry et al. (2006) after insertion of TE elements, segmental duplications and chromosomal rearrangements resulting from hybridization events. This is a more likely scenario to explain the observed regional triplication than invoking another round of polyploidization, followed by massive chromosome loss to return to the existing chromosome numbers of $2 n=16-20$, characteristic of the monogenomic Brassica species.

Acknowledgments We are indebted to Mr. Vincent D'Antonio and to Mrs. Fengliang Huang for technical assistance and to Dr. Roger Chetelat for his useful comments on the manuscript. The project was supported by the National Research Initiative of the USDA Cooperative State Research, Education and Extension Service, grant number to CFQ \# 2005-35301-15886 "Cloning and characterization of the major genes involved in the aliphatic glucosinolate pathway of Brassica crops".

Open Access This article is distributed under the terms of the Creative Commons Attribution Noncommercial License which permits any noncommercial use, distribution, and reproduction in any medium, provided the original author(s) and source are credited.

\section{References}

Alix K, Heslop-Harrison JS (2004) The diversity of retroelements in diploid and allotetraploid Brassica species. Plant Mol Biol 54:895-909

Altschul SF, Madden TL, Schaffer AA, Zhang J, Zhang Z, Miller W, Lipman DJ (1997) Gapped BLAST and PSI-BLAST: a new generation of protein database search programs. Nucleic Acids Res 25:3389-3402

Ayele M, Haas BJ, Kumar N, Wu H, Xiao Y (2005) Whole genome shotgun sequencing of Brassica oleracea and its application to gene discovery and annotation in Arabidopsis. Genome Res 15:487-495

Blanc G, Barakat A, Guyot R, Cooke R, Delseny M (2000) Extensive duplication and reshuffling in the Arabidopsis genome. Plant Cell 12:1093-1101

Burge C, Karlin S (1997) Prediction of complete gene structures in human genomic DNA. J Mol Biol 268:78-94

Flicek P, Keibler E, Hu P, Korf I, Brent MR (2003) Leveraging the mouse genome for gene prediction in human: from wholegenome shotgun reads to a global synteny map. Genome Res 13:46-54

Gao M, Li G, Yang B, McCombie WR, Quiros CF (2004) Comparative analysis of a Brassica BAC clone containing several major aliphatic glucosinolate genes with its corresponding Arabidopsis sequence. Genome 47:666-679

Gao M, Li G, McCombie WR, Quiros CF (2005) Comparative analysis of a transposon-rich Brassica oleracea BAC clone with its corresponding sequence in A. thaliana. Theor Appl Genet 111:949-955

Gao M, Li G, Potter D, McCombie WR, Quiros CF (2006) Comparative analysis of methylthioalkylmalate synthase (MAM) gene family and flanking DNA sequences in Brassica oleracea and A. thaliana. Plant Cell Rep 25:592-598

Henry Y, Bedhomme M, Blanc G (2006) History, protohistory and prehistory of the Arabidopsis thaliana chromosome complement. Trends Plant Sci 11:267-273

Hong CP, Plaha P, Koo DH, Yang TJ, Choi SR, Lee YK, Uhm T, Bang JW, Edwards D, Bancroft I, Park BS, Lee J, Lim YP (2006) A survey of the Brassica rapa genome by BAC-end sequence analysis and comparison with Arabidopsis thaliana. Mol Cells 22:300-307

Johnston JS, Pepper AE, Hall AE, Chen ZF, Hodnett G, Drabek J, Lopez R, Price HJ (2005) Evolution of genome size in Brassicaceae. Annals Bot 95:229-235

Katari MS, Balija V, Eilson RK, Martienssen RA, McCombie WR (2005) Comparing low coverage random shotgun sequence data from Brassica oleracea and Oryza stiva genome sequence for their ability to add to the annotation of Arabidopsis thaliana. Genome Res 15:496-504

Kowalski SP, Lan TH, Feldmann KA, Paterson AH (1994) Comparative mapping of Arabidopsis thaliana and Brassica oleracea chromosomes reveals islands of conserved organization. Genetics 138:499-510

Lagercrantz U (1998) Comparative mapping between Arabidopsis thaliana and Brassica nigra indicates that Brassica genomes have evolved through extensive genome replication accompanied by chromosome fusions and frequent rearrangements. Genetics 150:1217-1228

Lim YP, Plaha P, Choi SR, Uhm T, Hong CP, Bang JW, Hur YK (2006) Toward unraveling the structure of Brassica rapa genome. Physiol Plant 126(4):585-591

Lim KB, Yang TJ, Hwang YJ, Kim JS, Park JY, Kwon SJ, Kim J, Choi BS, Lim MH, Jin M, Kim HI, de Jong H, Bancroft I, Lim Y, Park BS (2007) Characterization of centromere and pericentromere retrotransposons in Brassica rapa and their distribution in related Brassica species. Plant J 49:173-183

Lysak MA, Koch MA, Pecinka A, Schubert I (2005) Chromosome triplication found across the tribe Brassiceae. Genome Res $15: 516-525$

O'Neill CM, Bancroft I (2000) Comparative physical mapping of segments of the genome of Brassica oleracea var. alboglabra that are homoeologous to sequenced regions of chromosomes 4 and 5 of Arabidopsis thaliana. Plant J 23:233-243

Park JY, Koo DH, Hong CP, Lee SJ, Jeon JW, Lee SH, Yun PY, Park BS, Kim HR, Bang JW, Plaha P, Bancroft I, Lim YP (2005) Physical mapping and microsynteny of Brassica rapa ssp. pekinensis genome corresponding to a $222 \mathrm{~kb}$ gene-rich region of Arabidopsis chromosome 4 and partially duplicated on chromosome 5. Mol Genet Genomics 274:579-588

Parkin IAP, Gulden SM, Sharpe AG, Lukens L, Trick M, Osborn TC, Lydiate DJ (2005) Segmental structure of the Brassica napus genome based on comparative analysis with Arabidopsis thaliana. Genetics 171:765-781

Rana D, van den Boogaart T, O’Neill CM, Hynes L, Bent E (2004) Conservation of the microstructure of genome segments in Brassica napus and its diploid relatives. Plant J 40:725-733

Schmidt R, Acarkan A, Boivin K, Clarenz O, Rossberg M (2003) The sequence of the Arabidopsis genome as a tool for comparative structural genomics in Brassicaceae. In: Nagata T, Tabata S 
(eds) Biotechnology in agricultural and forestry (BAF), vol 52. Springer, Heidelberg, pp 19-38

Schranz EM, Lysak MA, Mitchell-Olds T (2006) The ABC's of comparative genomics in the Brassicaceae: building blocks of crucifer genomes. Trends Plant Sci 11:1360-1385

Sikka SM (1940) Cytogenetics of Brassica hybrids and species. J Genet 40:441-509

Town CD, Cheung F, Maiti R, Crabtree J, Haas BJ, Wortman JR, Hine EE, Althoff R, Arbogast TS, Tallon LJ, Vigouroux M, Trick M, Bancroft I (2006) Comparative genomics of Brassica oleracea and Arabidopsis thaliana reveal gene loss, fragmentation, and dispersal after polyploidy. Plant Cell 18:1348-1359

Warwick SI, Black LD (1991) Molecular systematics of Brassica and allied genera (subtribe Brassicinae, Brassiceae)—chloroplast genome and cytodeme congruence. Theor Appl Genet 82:81-92

Wroblewski T, Coulibaly S, Sadowski J, Quiros CF (2000) Variation and phylogenetic utility of the Arabidopsis thaliana Rps2 homolog in various species of the tribe Brassiceae. Mol Phylogenet Evol 16:440-448
Yang YW, Lai KN, Tai PY, Li WH (1999) Rates of nucleotide substitution in angiosperm mitochondrial DNA sequences and dates of divergence between Brassica and the other angiosperm lineages. J Mol Evol 48:597-604

Yang TJ, Kim JS, Kwon SJ, Lim KB, Choi BS, Kim JA, Jin M, Park JY, Lim MH, Kim HI, Lim YP, Kang JJ, Hong JH, Kim CB, Bhak J, Bancroft I, Park BS (2006) Sequence-level analysis of the diploidization process in the triplicated FLOWERING LOCUS C region of Brassica rapa. Plant Cell 18:1339-1347

Zhang X, Wessler S (2004) Genome-wide comparative analysis of the transposable elements in the related species Arabidopsis thaliana and Brassica oleracea. PNAS 101:5585-5594

Ziolkowski PA, Kaczmarek M, Babula D, Sadowski J (2006) Genome evolution in Arabidopsis/Brassica: conservation and divergence of ancient rearranged segments and their breakpoints. Plant J 47:63-74 